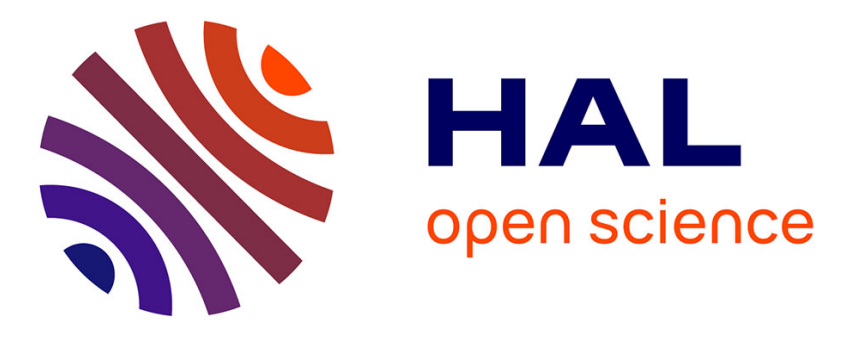

\title{
Enhancing with deconvolution the metrological performance of the grid method for in-plane strain measurement
}

Frédéric Sur, Michel Grediac

\section{- To cite this version:}

Frédéric Sur, Michel Grediac. Enhancing with deconvolution the metrological performance of the grid method for in-plane strain measurement. ICASSP - 38th International Conference on Acoustics, Speech, and Signal Processing, May 2013, Vancouver, Canada. pp.1563-1567, 10.1109/ICASSP.2013.6637914 • hal-00804719

\section{HAL Id: hal-00804719 https://hal.inria.fr/hal-00804719}

Submitted on 26 Mar 2013

HAL is a multi-disciplinary open access archive for the deposit and dissemination of scientific research documents, whether they are published or not. The documents may come from teaching and research institutions in France or abroad, or from public or private research centers.
L'archive ouverte pluridisciplinaire HAL, est destinée au dépôt et à la diffusion de documents scientifiques de niveau recherche, publiés ou non, émanant des établissements d'enseignement et de recherche français ou étrangers, des laboratoires publics ou privés. 


\section{ENHANCING WITH DECONVOLUTION THE METROLOGICAL PERFORMANCE OF THE GRID METHOD FOR IN-PLANE STRAIN MEASUREMENT}

\author{
Frédéric SUR \\ LORIA - projet Magrit \\ Université de Lorraine, CNRS, INRIA, France
}

\author{
Michel GRÉDIAC \\ Institut Pascal, IFMA, CNRS \\ Université Blaise Pascal, France
}

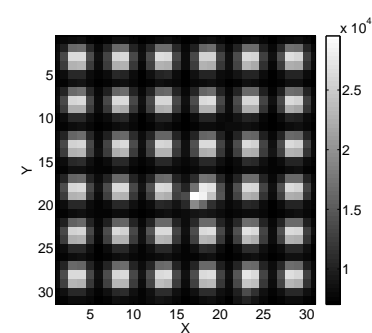

Fig. 1: Close-up of the grid on a deformed specimen [2].

- $f$ is the frequency of the carrier ;

- $\phi_{1}(x, y)$ and $\phi_{2}(x, y)$ are the carrier phase modulations due to specimen surface displacements along the $x-$ and $y$-axes respectively. In practice, their spatial derivatives are very small with respect to $f$.

This model proves to be accurate enough for our purposes. The displacement maps in the $x$ - and $y$-directions are actually proportional to $\Delta \phi_{1}$ and $\Delta \phi_{2}$ respectively, and the linearized strain components are linear combination of $\Delta \partial \phi_{1} / \partial x, \Delta \partial \phi_{1} / \partial y, \Delta \partial \phi_{2} / \partial x$, and $\Delta \partial \phi_{2} / \partial y$ [3], where $\Delta$ denotes the difference between the images before and after deformation. We thus focus here on the estimation of the phases $\phi_{1}$ and $\phi_{2}$ and their derivatives.

Sec. 2 is about the estimation of the phases and derivatives with windowed Fourier analysis. Sec. 3 characterizes how the digital noise in the grid images impairs the retrieved phases and phase derivatives. In Sec. 4, the estimations are enhanced with deconvolution algorithms. Sec. 5 is about related works.

\section{GRID METHOD AND CONVOLUTION}

The analysis of the grid is based on the windowed Fourier transform. Let us note:

$\Psi_{1}(\xi, \eta)=\iint_{\mathbb{R}^{2}} s(x, y) g_{\sigma}(x-\xi, y-\eta) e^{-2 i \pi f x} \mathrm{~d} x \mathrm{~d} y$
$\Psi_{2}(\xi, \eta)=\iint_{\mathbb{R}^{2}} s(x, y) g_{\sigma}(x-\xi, y-\eta) e^{-2 i \pi f y} \mathrm{~d} x \mathrm{~d} y$

where $g_{\sigma}$ is a 2D window function of width $\sigma$, symmetric, positive, and integrating to 1 . In practice, we use a Gaussian function of standard deviation $\sigma \geq 1 / f$ (which means that several lines lie inside the analysis window.) 
Without loss of generality, we study $\Psi_{1}$. It turns out that:

$$
\Psi_{1}(\xi, \eta) \simeq \frac{\gamma A}{2} d_{1} \iint g_{\sigma}(x-\xi, y-\eta) e^{i \phi_{1}(x, y)} \mathrm{d} x \mathrm{~d} y
$$

where $d_{1}$ is the first Fourier coefficient of $\ell$.

Let us give a heuristic justification of eq. (4). Plugging eq. (1) into (2), we can write $\Psi_{1}$ as the sum of:

- $A \iint g_{\sigma}(x-\xi, y-\eta) e^{-2 i \pi f x} \mathrm{~d} x \mathrm{~d} y$ of modulus $\left|\widehat{g_{\sigma}}(f, 0)\right| \simeq$ 0 assuming $\sigma \geq 1 / f{\text {. Indeed } \widehat{g_{\sigma}}}(f, 0)=e^{-2 \pi^{2} \sigma^{2} f^{2}}$.

- $\frac{\gamma A}{2} \iint \ell\left(2 \pi f x+\phi_{1}(x, y)\right) g_{\sigma}(x-\xi, y-\eta) e^{-2 i \pi f x} \mathrm{~d} x \mathrm{~d} y$. Using the Fourier series expansion $\ell\left(2 \pi f x+\phi_{1}(x, y)\right)=$ $\sum_{k \in \mathbb{Z}^{*}} d_{k} e^{i k\left(2 \pi f x+\phi_{1}(x, y)\right)}$, distributing the integral over the sum, and assuming that the variations of $\phi_{1}$ are negligible inside $g_{\sigma}$ and that $\iint g_{\sigma}(x-\xi, y-\eta) e^{2 i k^{\prime} \pi f x} \mathrm{~d} x \mathrm{~d} y \simeq 0$ (if $\left.k^{\prime} \neq 0\right)$ as above, then the integral amounts to $\frac{\gamma A}{2} d_{1} \iint g_{\sigma}(x-$ $\xi, y-\eta) e^{i \phi_{1}(x, y)} \mathrm{d} x \mathrm{~d} y$.

- $\frac{\gamma A}{2} \iint \ell\left(2 \pi f y+\phi_{2}(x, y)\right) g_{\sigma}(x-\xi, y-\eta) e^{-2 i \pi f x} \mathrm{~d} x \mathrm{~d} y$ which is $\simeq 0$ with the same arguments, assuming that the variations of $\phi_{2}$ are negligible inside the window $g_{\sigma}$.

Let us note "arg" the argument of any $z \in \mathbb{C} \backslash\{0\}, *$ the $2 \mathrm{D}$ convolution, and $\alpha=\arg \left(g_{\sigma} * e^{i \phi_{1}}\right)$. With eq. (4):

$$
\arg \left(\Psi_{1}\right)=\arg \left(d_{1}\right)+\alpha \bmod (2 \pi)
$$

Now, $\iint g_{\sigma}(x-\xi, y-\eta) e^{i\left(\phi_{1}(x, y)-\alpha(\xi, \eta)\right)} \mathrm{d} x \mathrm{~d} y=e^{-i \alpha(\xi, \eta)}$. $g_{\sigma} * e^{i \phi_{1}}(\xi, \eta)$ is a real number by definition of $\alpha$. Thus:

$$
\iint g_{\sigma}(x-\xi, y-\eta) \sin \left(\phi_{1}(x, y)-\alpha(\xi, \eta)\right) \mathrm{d} x \mathrm{~d} y=0
$$

Since the variations of $\phi_{1}$ inside $g_{\sigma}$ are small, then $\alpha(\xi, \eta) \simeq$ $\phi_{1}(x, y)$ in the preceding integral; hence a first order approximation of the sine $\left(\sin (x) \simeq x\right.$ ) yields (with $\iint g_{\sigma}=1$ ):

$$
\alpha(\xi, \eta) \simeq g_{\sigma} * \phi_{1}(\xi, \eta)
$$

We can conclude from eq. (5) and (7) that:

$$
\arg \left(\Psi_{1}\right)(\xi, \eta)=\arg \left(d_{1}\right)+g_{\sigma} * \phi_{1}(\xi, \eta) \quad \bmod (2 \pi)
$$

We have also, with $\cdot$ denoting either $\xi$ or $\eta$. :

$$
\frac{\partial \arg \left(\Psi_{1}\right)}{\partial \cdot}(\xi, \eta) \simeq g_{\sigma} * \frac{\partial \phi_{1}}{\partial \cdot}(\xi, \eta)
$$

A rigorous discussion of these results can be found in [4].

\section{TRANSFERRING THE IMAGE NOISE TO THE PHASE AND ITS DERIVATIVES}

Eq. (8) and (9) suggest to retrieve the actual phase $\phi_{1}$ and phase derivatives $\partial \phi_{1} / \partial$. with deconvolution. However, the grid image $s$ is impaired with noise, assumed here to be, for the sake of simplicity, an additive Gaussian white noise $n$

\footnotetext{
${ }^{1} \widehat{g}$ denotes here the Fourier transform of $g$
}

of variance $v$. This yields a noise process on the phase (denoted $\widetilde{n}$ ) and on the phase derivatives (denoted $\partial \widetilde{n} / \partial \cdot$ ), that we characterize in this section.

From now on, we use the discretized windowed Fourier transform. Since it is linear, in the presence of additive noise, $\Psi$ transforms into $\Psi_{n}=\Psi+\widehat{n}$ where:

$$
\widehat{n}(\xi, \eta)=\sum_{i, j} n\left(x_{i}, y_{j}\right) g_{\sigma}\left(x_{i}-\xi, y_{j}-\eta\right) e^{-2 i \pi f x_{i}} \Delta_{x} \Delta_{y}
$$

Here $\left(\Delta_{x}, \Delta_{y}\right)$ is the grid pitch (here $(1,1)$ pixel.)

A straightforward yet long calculation [4] proves that, if $\sigma \geq 1 / f$, then $\operatorname{Re}(\widehat{n})$ and $\operatorname{Im}(\widehat{n})$ are uncorrelated Gaussian variables and are both wide-sense stationary processes with covariance given by:

$$
\begin{aligned}
\operatorname{Covar}(\operatorname{Re} & \left.(\widehat{n}(\xi, \eta)), \operatorname{Re}\left(\widehat{n}\left(\xi^{\prime}, \eta^{\prime}\right)\right)\right) \\
= & \operatorname{Covar}\left(\operatorname{Im}(\widehat{n}(\xi, \eta)), \operatorname{Im}\left(\widehat{n}\left(\xi^{\prime}, \eta^{\prime}\right)\right)\right) \\
& =\frac{v \Delta_{x} \Delta_{y}}{8 \pi \sigma^{2}} e^{-\left(\xi-\xi^{\prime}\right)^{2} /\left(4 \sigma^{2}\right)-\left(\eta-\eta^{\prime}\right)^{2} /\left(4 \sigma^{2}\right)}
\end{aligned}
$$

\subsection{Noise on the phase}

Since $\arg \left(\Psi_{n}\right)=\arctan \left(\frac{\operatorname{Im}(\Psi)+\operatorname{Im}(\widetilde{n})}{\operatorname{Re}(\Psi)+\operatorname{Re}(\widetilde{n})}\right)$, a first order Taylor expansion yields:

$$
\begin{array}{r}
\arg \left(\Psi_{n}\right)(\xi, \eta)=\arg (\Psi)(\xi, \eta)-\frac{\operatorname{Im}(\Psi(\xi, \eta, 0))}{|\Psi(\xi, \eta, 0)|^{2}} \operatorname{Re}(\widehat{n})(\xi, \eta) \\
+\frac{\operatorname{Re}(\Psi(\xi, \eta, 0))}{|\Psi(\xi, \eta, 0)|^{2}} \operatorname{Im}(\widehat{n})(\xi, \eta)
\end{array}
$$

Hence, $\widetilde{n}$ is approximately a 0 -mean spatially-correlated Gaussian random variable. With the properties of $\widehat{n}$ and eq. (11), it is possible to compute the autocovariance of $\widetilde{n}$ [4]:

$$
\begin{array}{r}
\operatorname{Covar}\left(\widetilde{n}(\xi, \eta), \widetilde{n}\left(\xi^{\prime}, \eta^{\prime}\right)\right)=\frac{v \Delta_{x} \Delta_{y}}{8 \pi \sigma^{2}\left|\Psi_{1}(\xi, \eta)\right|\left|\Psi_{1}\left(\xi^{\prime}, \eta^{\prime}\right)\right|} \\
\cdot e^{-\left(\xi-\xi^{\prime}\right)^{2} /\left(4 \sigma^{2}\right)-\left(\eta-\eta^{\prime}\right)^{2} /\left(4 \sigma^{2}\right)}
\end{array}
$$

Now, for the same reason that gives eq. (6): $\left|\Psi_{1}(\xi, \eta)\right| \simeq$ $\left|d_{1}\right| \frac{\gamma A}{2} \iint g_{\sigma}(x-\xi, y-\eta) \cos \left(\phi_{1}(x, y)-\alpha_{\sigma}(\xi, \eta)\right) \mathrm{d} x \mathrm{~d} y$. Hence $\cos \simeq 1$, and $\left|\Psi_{1}(\xi, \eta)\right| \simeq\left|d_{1}\right| \frac{\gamma A}{2}$ is a constant, denoted by $K$ in the remainder of the article.

Consequently, the noise $\tilde{n}$ on the phase map can be considered as a stationary spatially correlated process.

\subsection{Noise on the phase derivatives}

With the same assumption as above, the noise process $\partial \widetilde{n} / \partial$. on the phase derivative is a stationary spatially correlated process, whose autocovariance function is the opposite of the second derivative of the autocovariance of $\widetilde{n}[5]$ :

$$
\begin{gathered}
\operatorname{Covar}\left(\frac{\partial \widetilde{n}}{\partial \xi}(\xi, \eta), \frac{\partial \widetilde{n}}{\partial \xi}\left(\xi^{\prime}, \eta^{\prime}\right)\right)=\frac{v \Delta_{x} \Delta_{y}}{16 \pi \sigma^{4} K^{2}} \\
\cdot e^{-\left(\xi-\xi^{\prime}\right)^{2} /\left(4 \sigma^{2}\right)-\left(\eta-\eta^{\prime}\right)^{2} /\left(4 \sigma^{2}\right)}\left(1-\frac{\left(\xi-\xi^{\prime}\right)^{2}}{2 \sigma^{2}}\right)
\end{gathered}
$$




$$
\begin{gathered}
\operatorname{Covar}\left(\frac{\partial \widetilde{n}}{\partial \eta}(\xi, \eta), \frac{\partial \widetilde{n}}{\partial \eta}\left(\xi^{\prime}, \eta^{\prime}\right)\right)=\frac{v \Delta_{x} \Delta_{y}}{16 \pi \sigma^{4} K^{2}} \\
\cdot e^{-\left(\xi-\xi^{\prime}\right)^{2} /\left(4 \sigma^{2}\right)-\left(\eta-\eta^{\prime}\right)^{2} /\left(4 \sigma^{2}\right)}\left(1-\frac{\left(\eta-\eta^{\prime}\right)^{2}}{2 \sigma^{2}}\right)
\end{gathered}
$$

\section{DECONVOLUTION FOR THE GRID METHOD}

The problem is to retrieve $\phi_{1}$ (resp. its derivatives) from $\arg \left(\Psi_{n}\right)$ (resp. its derivatives) thanks to:

$$
\begin{aligned}
\arg \left(\Psi_{n}\right) & =\arg \left(d_{1}\right)+g_{\sigma} * \phi_{1}+\widetilde{n} \\
\frac{\partial \arg \left(\Psi_{n}\right)}{\partial \cdot} & =g_{\sigma} * \frac{\partial \phi_{1}}{\partial \cdot}+\frac{\partial \widetilde{n}}{\partial \cdot}
\end{aligned}
$$

In both cases, this non-blind deconvolution problem writes: $u=g_{\sigma} * u_{0}+\bar{n}$. We assess here four popular deconvolution algorithms [6] (giving an approximation $u^{\prime}$ of $u_{0}$ ), together with the estimate which we deconvolve, namely:

1. Direct deconvolution, noted DD, where $u^{\prime}$ is obtained by inverse Fourier transform and: $\widehat{u^{\prime}}=\widehat{u} / \widehat{g_{\sigma}}$.

2. Regularized (Tikhonov) deconvolution, noted RD, where $u^{\prime}$ minimizes $\left\|g_{\sigma} * u^{\prime}-u\right\|_{2}^{2}+\lambda\left\|\Delta u^{\prime}\right\|_{2}^{2}$.

3. Richardson-Lucy algorithm. Since it works on nonnegative images, we test two possible workarounds: 1) RL is run on $\exp (u)$ and $u^{\prime}$ is the logarithm of the result (noted $\mathrm{RL} 1)$; 2) RL is run on $u-\min (u)$ and $u^{\prime}$ is normalized so that its mean equal to the mean of $u$ (noted RL2.)

4. Wiener filtering, which needs as input the mean power spectral density (PSD) $M$ of the random noise $\bar{n}$ and the power spectral density $S$ of $u_{0}$. We test two possible estimations for $M: 1)$ simplifying $\bar{n}$ in a white noise whose variance is given by imposing $\xi=\xi^{\prime}$ and $\eta=\eta^{\prime}$ in eq. (13) (for phase restoration) or eq. (14-15) (for phase derivative restoration), giving a constant $M(x, y)$ equal to this variance (method noted WF1); 2) $M(x, y)$ is from Wiener-Khinchin theorem the Fourier transform of either eq. (13) or eq. (14-15) (noted WF2.) In both cases $S$ is estimated as the PSD of $u$.

5. the classic estimate of the literature $[1,7]$ which consists in estimating the phase and the derivatives directly as $\arg \left(\Psi_{n}\right)$ or $\partial \arg \left(\Psi_{n}\right) / \partial$., without any deconvolution (noted CE.)

In spite that WF2 is the only method that uses a realistic assumption on noise, we still test the other ones, as e.g. in [8] where RL is used to enhance spectrograms. We use the implementation provided by the Matlab image processing toolbox. Here, experiments are presented on synthetic data. Additional experiments on asphalt specimens and shape memory alloys and further discussion are provided in [9].

A synthetic grid image is generated following eq. (1), with $A=2^{11}, \gamma=0.9, f=1 / 5$, and $\ell(x)=\sin (x)^{3}$. These values are realistic compared to the specimen of interest. Such a $\ell$ is chosen to simulate sharp lines and to illustrate that a pure sine is not necessarily the spatial carrier function; in this case $d_{1}=-3 i / 8$. The phase maps $\phi_{1}$ and $\phi_{2}$ are here synthetic images depicted in Fig. 2, normalized such that the derivatives (estimated with finite difference scheme) along $\xi$ and $\eta$ axis are below 0.001 (still a realistic value). While $\phi_{1}$ is smooth, $\phi_{2}$ is not. The synthetic grid image is then quantized over 12 bits, and noise standard deviation varies between 0 and 4 (with the Sensicam QE-one camera employed in real experiments, $\sqrt{v}=2$ seems to be realistic.) Note that some information is irremediably lost by quantization, hence the phases and phase derivatives cannot be perfectly retrieved.

Fig. 3 assesses the validity of the approximations given by eq. (8) and (9). It shows the Normalized Root Mean Square Error (NRMSE) between the estimation of the phases $\arg \left(\Psi_{n}\right)$ or of their derivatives, and of the true value convolved with $g_{\sigma}$, for several noise variances $v$ and sizes $\sigma$ of the window. Note that the approximation is valid up to $1-5 \%$ for realistic values of the noise, and that it gets tighter as $\sigma$ increases.

Fig. 4 shows the NRMSE (estimated over a central area covering $70 \%$ of the map to get rid of ringing artifacts on the image edges) between the deconvolved phase or derivative maps and the true values, for several $v$ and $\sigma=5$ or 9 . Large noise levels make some methods fail, yielding large NRMSE. The phase estimation always benefits from deconvolution (compare to CE) for realistic levels of noise, the NRMSE being divided by 2 to 10 . Concerning the phase derivatives, WF2 outperforms all other methods, especially for a large noise level. It permits to retrieve the real derivative with NRMSE $<10 \%$ for realistic noise levels, and improves over CE. RL1 performs well in many cases. When $\sigma$ grows, the advantage of WF2 decreases as noise is smoothed out. Retrieving $\partial \phi_{2} / \partial \eta$ is difficult because of the singularities.

Fig. 5 shows deconvolution results of RL1 and WF2, superimposed on the actual value $\partial \phi / \partial$. (solid blue line) and the value estimated by CE (solid green line). We only show results about the phase derivatives. Here $\sqrt{v}=4$ and $\sigma=5$. On these cross-sections along $\xi=700$, we can see that the spatially correlated noise yields sinusoidal waves on the green line, which are accentuated by RL1 (in red). On the contrary, WF2 diminishes the effect of these noise patterns, and gives a more accurate estimation of the blue line.

\section{RELATION TO PRIOR WORK}

Another full-field measurement technique used in experimental solid mechanics is Digital Image Correlation [10], which is based on transferring random marking on the specimen surface instead of a regular grid. To the best of our knowledge, no comparative study of the metrological performances is available. Various techniques are available for processing grid images [11], among which the most popular ones are based on Windowed Fourier Transform [1, 7]. The proposed contribution is to enhance the grid method with deconvolution, and to characterize the correlated noise on the phases and the phase derivatives. This study is also of interest for fringe pattern analysis [12] since grid analysis is a special case of it. 

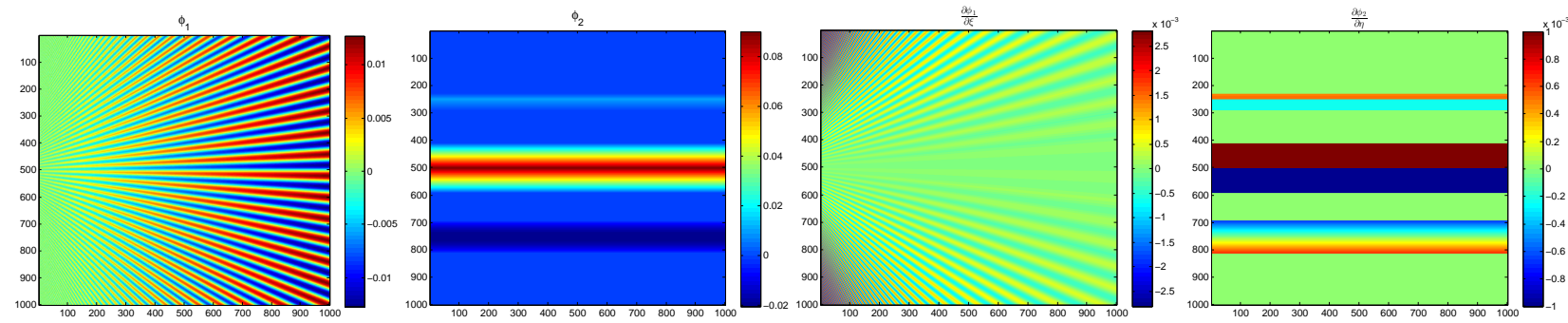

Fig. 2: Synthetic phases $\phi_{1}$ and $\phi_{2}$, and phase derivatives $\partial \phi_{1} / \partial \xi$ and $\partial \phi_{2} / \partial \eta$.
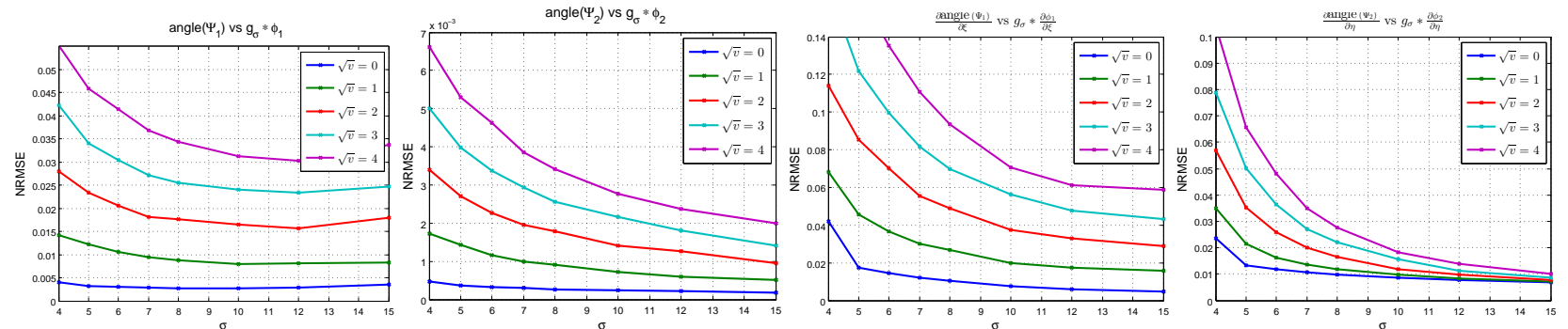

Fig. 3: Assessing the accuracy of the approximations given by eq. (8) and (9).
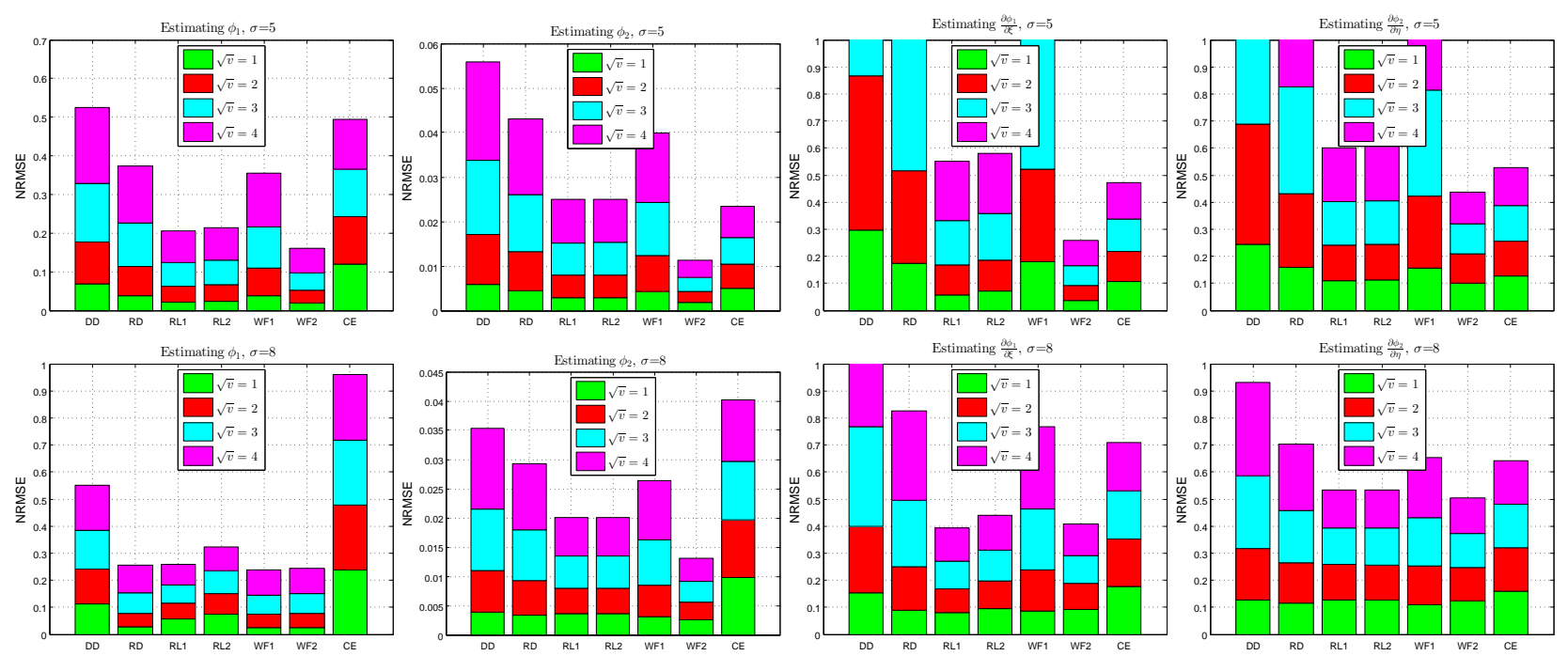

Fig. 4: Comparison of the deconvolution performance of several algorithms.
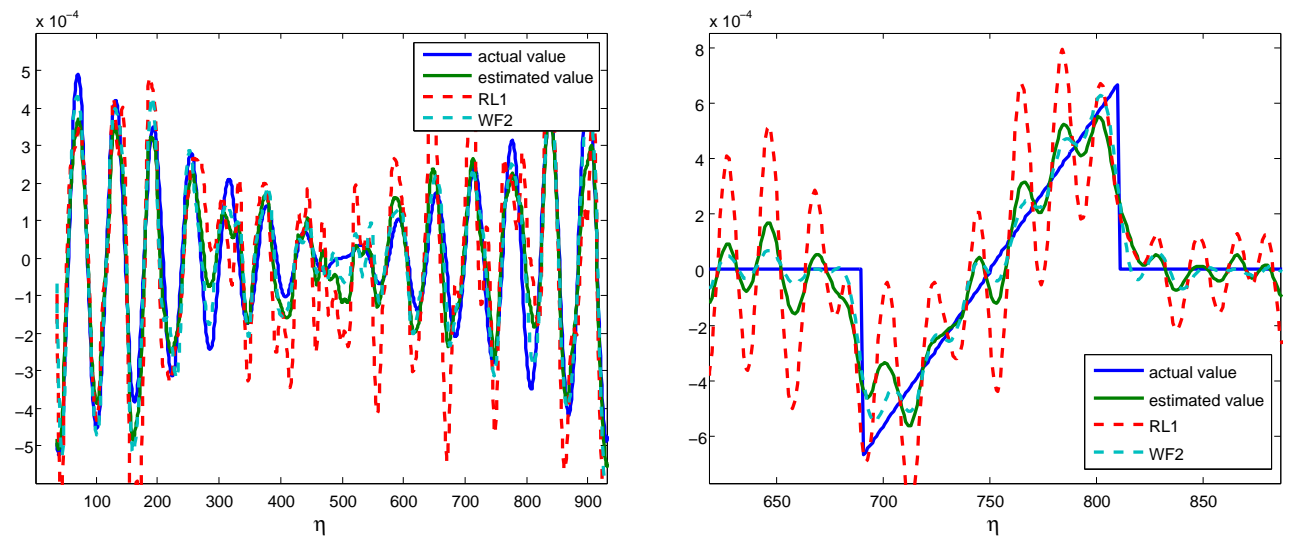

Fig. 5: Examples of restoration of $\partial \phi_{1} / \partial \xi$ (on the left) and of $\partial \phi_{2} / \partial \eta$ (close-up on the right.) 


\section{REFERENCES}

[1] C. Badulescu, M. Grédiac, and J.-D. Mathias, "Investigation of the grid method for accurate in-plane strain measurement," Measurement Science and Technology, vol. 20, no. 9, pp. 095102, 2009.

[2] M. Grédiac and E. Toussaint, "Studying the mechanical behaviour of asphalt mixtures with the grid method," Strain. An International Journal for Experimental Mechanics, vol. 49, no. 1, pp. 1-15, 2013, Wiley.

[3] T.M. Atanackovic and A. Guran, Theory of Elasticity for Scientists and Engineers, Springer, 2000.

[4] F. Sur and M. Grédiac, "Towards deconvolution to enhance the grid method for in-plane strain measurement," Research Report 8126, INRIA, November 2012.

[5] P. Abrahamsen, "A review of Gaussian random fields and correlation functions," Tech. Rep., Norwegian Computing Center, Oslo, 1997.

[6] R.C. Gonzalez and R.E. Woods, Digital Image Processing (3rd Edition), Prentice-Hall, 2006.

[7] Y. Surrel, Photomechanics, vol. 77 of Topics in Applied Physics, chapter Fringe analysis, pp. 55-102, Springer, 2000.

[8] W.-K. Lu and Q. Zhang, "Deconvolutive short-time Fourier transform spectrogram," IEEE Signal Processing Letters, vol. 16, no. 7, pp. 576-579, 2009.

[9] M. Grédiac, F. Sur, C. Badulescu, and J.-D. Mathias, "Using deconvolution to improve the metrological performance of the grid method," Optics and Lasers in Engineering, vol. 51, no. 6, pp. 716-734, 2013.

[10] M. Sutton, J.-J. Orteu, and H. Schreier, Image Correlation for Shape, Motion and Deformation Measurements, Springer, 2009.

[11] C. Badulescu, M. Bornert, J.-C. Dupré, S. Equis, M. Grédiac, J. Molimard, P. Picart, R. Rotinat, and V. Valle, "Demodulation of spatial carrier images: performance analysis of several algorithms," Experimental Mechanics, 2013, in press.

[12] Q. Kemao, “Two-dimensional windowed Fourier transform for fringe pattern analysis: Principles, applications and implementations," Optics and Lasers in Engineering, vol. 45, no. 2, pp. 304-317, 2007. 